\title{
The simulation of cable harness based on mass-spring model
}

\author{
Yuan Bin ${ }^{1, a}$, Du Hongwang ${ }^{1}$, Wang Haitao ${ }^{1}$, Xiong wei ${ }^{1}$ \\ ${ }^{1}$ Ship Electromechanical Equipment Institute, Dalian Maritime University, CN
}

\begin{abstract}
The simulation of cable harnesses is challenging due to the complex structure and the large deformation during the assembly operations. In this paper an improved mass-spring model is approached to simulate the shape of cable harnesses and to calculate the deformation of cable harnesses. Based on a virtual software platform Open Cascade and using Newmark- $\beta$ algorithm which could reduce the instabilities of equation, the simulation is completed. Then, a cable geometry measurement bench with the 3D laser scan technology is construed to verify the accuracy of the algorithm. The result shows the model could simulate the deformation very well.
\end{abstract}

\section{Introduction}

With the development of electromechanical products to be more integrated, lightweight, cable harness which connects two electrical equipments has more and more application. And the cable design and assembly also have become an important factor during the all life cycle for electromechanical products [1]. The early manufacturing and installation methods for cable harness are handmade or computer aided design cabling. The handmade method has a low efficiency, a long production cycle and high cost. While the CAD cabling bases on the cable design drawing, the cable key position points are defined and the digital model is gained through interpolation fitting. In this way, cables are operated as rigid bodies; the bending is implemented by constant bending radius. And the result is the design length of cable is usually too long, which increase the weight of the product and change the centroid that is very adverse scenarios for the product. Therefore simulation the assembly of cable harnesses with virtual assembly has become a new and necessary way. With virtual reality technology, cable designer could determine the cable route path, branch positions and bundle schemes based on the virtual prototype. And a rational and accurate cable model could be gained [2]. The research about the simulation of the cable harness has been developed for long time. George C [3] and Huang M J [4] both used a spline curve to fit the cable harness. They computed the potential energy of the curve. And they got the configuration of the cable when the potential energy is minimums. A.N.F. Klimowicz [5] use FEM method to simulate the deformation of cable harnesses. In this way, cables were divided into many meshes. Interpolating functions is used to calculate the stress and strain between adjacent meshes. This method was very accurately for the simulation, while the time cost is high.

\section{Modelling of the cable harness}

\subsection{Mass-spring model.}

Mass-Spring system is proposed originally by Provot [6] used for cloth simulation. This method assumed the soft objects is the collection of a number of mass points which is connected through three kinds of linear springs. As shown in Figure1. Lock first used mass-spring system in cable harness [7]. Our work is also based on this method.

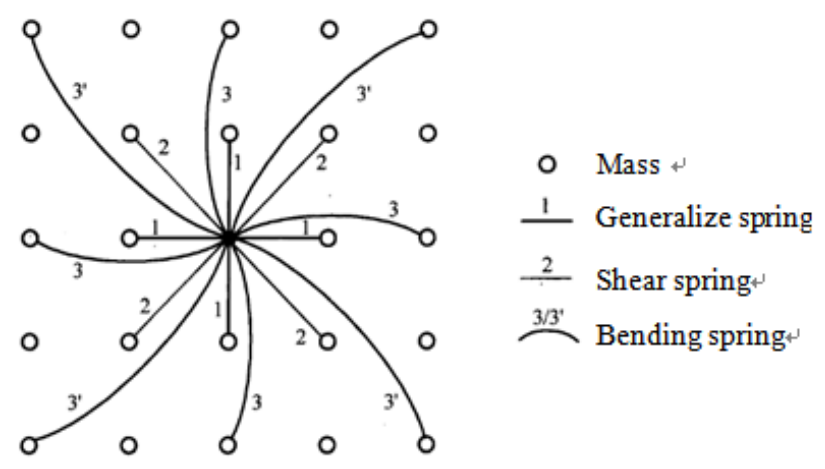

Figure 1. Mass-spring model

In our model, cable is thought as some discrete masses which are connected with springs while the anisotropism of the cable is omitted. The model includes three types of spring: structural spring, bending spring, and the leading spring. As shown in Figure2. Structural spring preserves the length of cable during the motion. The bending spring will shown the bending characteristics, while the leading spring will represent the effect of the plug and bandage. According to [7] the motion of the masses is determined

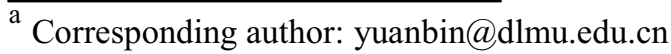


by the potential energy $\boldsymbol{E}$ of the spring, and the massspring system obtain the Newton's second law.

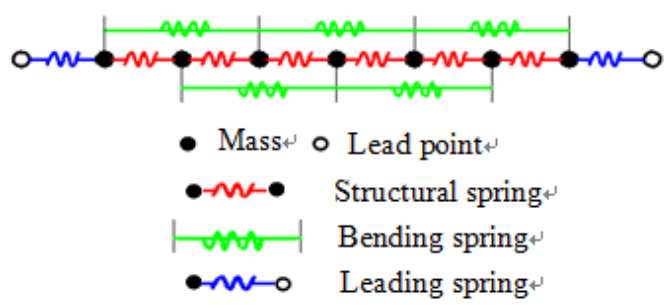

Figure 2. The model of cable

$$
\ddot{\mathbf{X}}=\mathbf{M}^{1}\left(-\frac{\partial \boldsymbol{E}}{\partial \mathbf{X}}+\mathbf{F}\right)
$$

The configuration of cable harness is defined by the position of mass points. Resolving this equation could get the stable configuration.

\subsection{The Definition of Spring}

\subsubsection{Structural Spring}

Structural spring connecting the two successive points will preserve the length of cable. The energy function for the structural spring is

$$
\boldsymbol{E}_{i}(\mathbf{x})=\frac{k_{s}}{2}\left(\left|\mathbf{x}_{i}-\mathbf{x}_{i-1}\right|-l\right)\left(\left|\mathbf{x}_{i}-\mathbf{x}_{i-1}\right|-l\right)
$$

Where $k_{s}$ is the elastic modulus of the structural spring, and $l$ is the natural length. The restoring forces acting on the $i$ th particle as the first derivative of the energy is

$$
\begin{aligned}
\boldsymbol{f}_{1 i}(\mathbf{x}) & =-\frac{\partial \boldsymbol{E}}{\partial \mathbf{X}}=-\left(\frac{\partial \boldsymbol{E}_{i}}{\partial \mathbf{X}_{i}}+\frac{\partial \boldsymbol{E}_{i+1}}{\partial \mathbf{X}_{i}}\right) \\
& =k_{s}\left(\frac{l}{\left|\mathbf{X}_{i}-\mathbf{X}_{i-1}\right|}-1\right)\left(\mathbf{X}_{i}-\mathbf{X}_{i-1}\right) \\
& -k_{s}\left(\frac{l}{\left|\mathbf{X}_{i+1}-\mathbf{X}_{i}\right|}-1\right)\left(\mathbf{x}_{i+1}-\mathbf{X}_{i}\right)
\end{aligned}
$$

The damping spring is designed to show the internal damping of the cable during the configuration simulation. And the damping force is:

$$
\begin{aligned}
\boldsymbol{f}_{d i}(\mathbf{X}) & =-k_{d} \frac{\left(\mathbf{X}_{i}-\mathbf{X}_{i-1}\right)\left(\mathbf{X}_{i}-\mathbf{X}_{i-1}\right)^{T}}{\left|\mathbf{X}_{i}-\mathbf{X}_{i-1}\right|^{2}}\left(\mathbf{v}_{i}-\mathbf{v}_{i-1}\right) \\
& +k_{d} \frac{\left(\mathbf{X}_{i+1}-\mathbf{X}_{i}\right)\left(\mathbf{X}_{i+1}-\mathbf{X}_{i}\right)^{T}}{\left|\mathbf{X}_{i+1}-\mathbf{X}_{i}\right|^{2}}\left(\mathbf{v}_{i+1}-\mathbf{v}_{i}\right)
\end{aligned}
$$

\subsubsection{Bending Spring}

Bending spring is a linear spring connecting the $i$ th mass point with the $(i+2)$ th point used to describe the elastic bending behavior, as the dashed line shown in Figure 3.

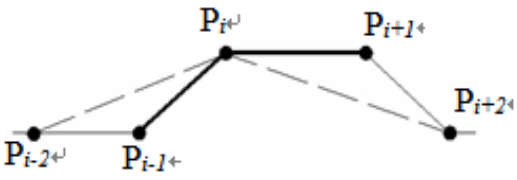

Figure 3. The bengding spring

The energy of the bending spring in terms of the positions of the mass points is:

$$
\boldsymbol{E}_{i}(\mathbf{x})=\frac{k_{b}}{2}\left(\left|\mathbf{x}_{i}-\mathbf{x}_{i-2}\right|-2 l\right)\left(\left|\mathbf{x}_{i}-\mathbf{x}_{i-2}\right|-2 l\right)
$$

Where $k_{b}$ is the elastic modulus of the bending spring. The restoring forces acting on the $x_{i}$ particle as the first derivative of the energy is

$$
\begin{aligned}
\boldsymbol{f}_{2 i}(\mathbf{x}) & =-\frac{\partial \boldsymbol{E}}{\partial \mathbf{x}}=-\left(\frac{\partial \boldsymbol{E}_{i}}{\partial \mathbf{X}_{i}}+\frac{\partial \boldsymbol{E}_{i+2}}{\partial \mathbf{X}_{i}}\right) \\
& =k_{b}\left(\frac{l}{\left|\mathbf{X}_{i}-\mathbf{X}_{i-2}\right|}-1\right)\left(\mathbf{X}_{i}-\mathbf{X}_{i-2}\right) \\
& -k_{b}\left(\frac{l}{\left|\mathbf{X}_{i+2}-\mathbf{X}_{i}\right|}-1\right)\left(\mathbf{X}_{i+2}-\mathbf{X}_{i}\right)
\end{aligned}
$$

\subsubsection{Leading Spring}

In assembly product, the shape of cable harness is affected by the plug and bandage. A leading spring is assumed as the plug to constraint the position of mass. Leading spring is similar with the bending spring; however the stiffness is much greater. The restoring force is proportional in angle, as shown in Figure 4 Where the $i$ th mass is a fixed point and the $i+1$ th mass is a movable point.

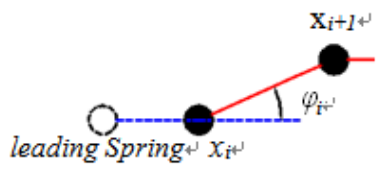

Figure 4. The leadding spring

The energy of the leaning spring is given by following formula, where $k_{l}$ is the elastic modulus of the leading spring:

$$
\boldsymbol{E}_{i}(\mathbf{x})=\frac{k_{l}}{2} \varphi_{i}^{2}
$$

In order to simplify the expressions we set $\mathbf{a}_{i}=\left(\mathbf{x}_{i+l}-\mathbf{x}_{i}\right)$, and $\mathbf{b}$ is the unit vector of the direction of leading spring, therefore the angle can be expressed as:

$$
\varphi_{i}=\frac{k_{l}}{2}\left[\tan ^{-1}\left(\frac{\left|\mathbf{a}_{i} \times \mathbf{b}\right|}{\mathbf{a}_{i} \cdot \mathbf{b}}\right)\right]^{2}
$$


Leading spring only works on the points beside the fix points. The restoring forces acting on the $i$ th particle which is after the fixed points is:

$$
\begin{aligned}
& \boldsymbol{f}_{3 i}(\mathbf{x})=-\frac{\partial \boldsymbol{E}_{i-1}}{\partial \mathbf{x}_{i}}=-k_{l} \varphi_{i-1} \frac{\partial \varphi_{i-1}}{\partial \mathbf{a}_{i-1}} \\
& =k_{l} \varphi_{i-1} \frac{\mathbf{a}_{i-1} \times\left(\mathbf{a}_{i-1} \times \mathbf{b}\right)}{\mathbf{a}^{2}\left|\mathbf{a}_{i-1} \times \mathbf{b}\right|}
\end{aligned}
$$

Meanwhile, the restoring forces acting on the $i$ th particle which is before the fixed points is:

$$
\boldsymbol{f}_{3 i}(\mathbf{x})=-\frac{\partial \boldsymbol{E}_{i+1}}{\partial \mathbf{x}_{i}}=k_{l} \phi_{i+1} \frac{\mathbf{a}_{i+1} \times\left(\mathbf{a}_{i+1} \times \mathbf{b}\right)}{\mathbf{a}^{2}{ }_{i+1}\left|\mathbf{a}_{i+1} \times \mathbf{b}\right|}
$$

\section{Computation of the Equation}

We adopted the Newmark- $\beta$ method which is used to resolve structural dynamics in blast wave or seismic load [8] to compute the differential equation. Newmark- $\beta$ method is an implicit method that is possible to improve the stability.

According to the Newmark- $\beta$ method, the position and the velocity of $i$ th point mass on time $t$ can be obtain by the following formulas:

$$
\left\{\begin{array}{l}
\mathbf{x}_{t}=\mathbf{x}_{t+\Delta t}+\Delta t \cdot \dot{\mathbf{x}}_{t+\Delta t}+\frac{1}{4} \Delta t^{2}\left(\ddot{\mathbf{x}}_{t+\Delta t}+\ddot{\mathbf{x}}\right) \\
\dot{\mathbf{x}}_{t}=\dot{\mathbf{x}}_{t+\Delta t}+\frac{1}{2} \Delta t^{2}\left(\ddot{\mathbf{x}}_{t+\Delta t}+\ddot{\mathbf{x}}\right)
\end{array}\right.
$$

Based on the equation from1-10, the motion equation of cable could be deduced as a second-order differential equation set:

$$
\left\{\begin{array}{l}
\boldsymbol{f}_{i x}\left(\ddot{\mathbf{x}}_{i}, \ddot{\mathbf{y}}_{i}, \ddot{\mathbf{z}}_{i}\right)=0 \\
\boldsymbol{f}_{i y}\left(\ddot{\mathbf{x}}_{i}, \ddot{\mathbf{y}}_{i}, \ddot{\mathbf{z}}_{i}\right)=0 \\
\boldsymbol{f}_{i z}\left(\ddot{\mathbf{x}}_{i}, \ddot{\mathbf{y}}_{i}, \ddot{\mathbf{z}}_{i}\right)=0
\end{array}\right.
$$

The integration methods of an ordinary differential equation include explicit methods and implicit methods. Equation 12 is solved with Newton iteration. And acceleration in three coordinate axes is gotten. Then put acceleration in equation 11 to get the numerical solution of position and velocity. The shape of the cable harness is obtained by repeating the above calculations until the velocity becomes smaller than some specified threshold. Therefore the shape of cable could be simulated.

\section{Experimental Verification}

In order to simulate the configuration of cable based on the above model, a simulation platform is edited based on Open Cascade (OCC) software. In the platform, the calculation result of above model is connected with a Bspline curve. And then with the ::onPipe() command, the configuration of cable is realized by lofting a circle shape section on that curve. The Simulation result is given in Figure 5 with the condition is that the distance between the two ends is $70 \mathrm{~cm}$, angle between the directions of two ends is 60 degree, and the height difference is $30 \mathrm{~cm}$. And the other parameters are shown in Table1.

Table1: The Value of Parameters

\begin{tabular}{|c|c|}
\hline Parameters & Value \\
\hline length $(\mathrm{cm})$ & 100 \\
\hline segments & 20 \\
\hline$k_{s} / m$ & $4.75 \cdot 10^{3}$ \\
\hline$k_{b} / m$ & $5 \cdot 10^{2}$ \\
\hline$k_{d} / m$ & $2.5 \cdot 10^{3}$ \\
\hline$k_{l} / m$ & $3 \cdot 10^{3}$ \\
\hline
\end{tabular}

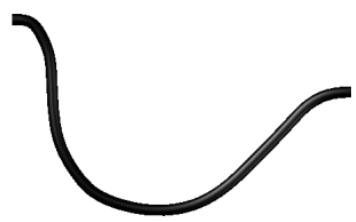

Figure 5. Simulation of cable

To verify the correctness of the mass-spring model and calculation algorithm, an experimental bench is constructed based on 3D laser scan technology, as shown in Figure 6. The bench includes an aluminum base, clamp mechanism, articulated arm laser scanner and a pc. Clamp mechanism includes straight rod, cross universal joint and rod holder. The position and angle of cable's two-end could be gotten through the graduation in the holder. We use Geomagic Qualify, the reverse engineering software, to process the scanning data, and then the $3 \mathrm{D}$ geometry configuration could be gained.

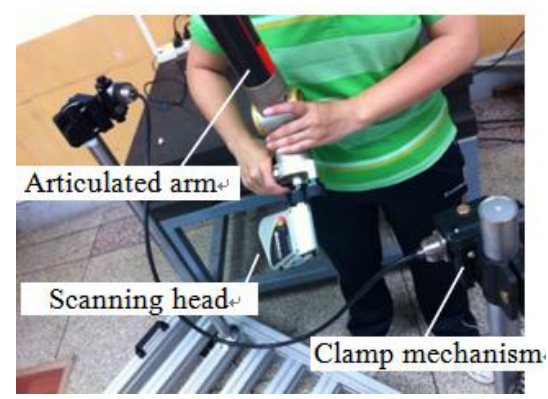

Figure 6. Configuration of cable

\section{Conclusion}

In this paper, the simulation of cable harness is complete based on an improved mass-spring model. Newmark- $\beta$ method is used to get spatial deformable of cable geometry configuration. Moreover, the actual geometry configuration of cable is achieved with $3 \mathrm{D}$ scanning technology. Through comparison, the accuracy of the model has been verified. In the future, we mainly focus on the design of the parameters of the different kinds of spring. 


\section{Acknowledgement}

The work in this paper is supported by the Fundamental Research Funds for the Central Universities (3132014065) and the Dalian Maritime University Projects (0039101408).

\section{References}

1. Wan B L. Research on Cable Harness Wiring Design and Assembly Process Planning Technology in Virtual Environment [D]. Beijing of China: Beijing Institute of Technology, 2007.

2. NG F M, Ritchie, J. M., Simmons, J. E. L. The Design and Planning of Cable Harness Assemblies [J], Journal of Materials Processing Technology, (2000), 107: 37 - 43

3. George C, Dave G. Deformable Curve and Surface Finite-Elements for Free-Form Shape Design [C] // Proceedings of the 18th annual conference on Computer graphics and interactive techniques (S089791-436-8), 1991, 25(4):257-266.
4. Huang M J, Dong X P. Research on Flexible Cable Geometric Modeling Technology in Virtual Maintenance Based on VRML [C]. 2010 International Conference on Mechanic Automation and Control Engineering, 2010, 772-775.

5. Klimowicz ANF, Mihajlovic, MD. Modelling 3D Semi-deformable Tubes in Real Time [C]. // International Conference of Computational Methods in Sciences and Engineering (S0096-3003), 2007, 184(1):52-62.

6. X. Provot. Deformation constraints in a mass-spring model to describe rigid cloth behaviour[J]. Graphics Interface, 1995, 33(3), 147-154

7. Achim L, Elmar S. A Virtual Environment for Interactive Assembly Simulation:From Rigid Bodies to Deformable Cables [C]. // 5th World Multiconference on Systemics, Cybernetics and Informatics, 2001: 325-332.

8. Edward L. Wilson, Static \& Dynamic Analysis of Structures [M], China Architecture \& Building Press, (2006) (in Chinese) 